\title{
Cerebellum-specific and age-dependent expression of an endogenous retrovirus with intact coding potential
}

Kang-Hoon Lee ${ }^{1,3}$, Makoto Horiuchi ${ }^{2,3}$, Takayuki Itoh ${ }^{2,3}$, David G Greenhalgh ${ }^{1,3}$ and Kiho Cho ${ }^{1,3^{*}}$

\begin{abstract}
Background: Endogenous retroviruses (ERVs), including murine leukemia virus (MuLV) type-ERVs (MuLV-ERVs), are presumed to occupy $\sim 10 \%$ of the mouse genome. In this study, following the identification of a full-length MuLVERV by in silico survey of the C57BL/6J mouse genome, its distribution in different mouse strains and expression characteristics were investigated.

Results: Application of a set of ERV mining protocols identified a MuLV-ERV locus with full coding potential on

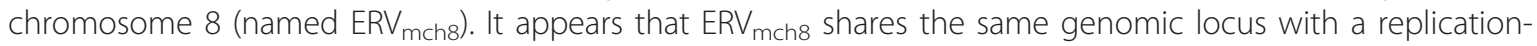
incompetent MuLV-ERV, called Emv2; however, it was not confirmed due to a lack of relevant annotation and Emv2 sequence information. The ERV ${ }_{\text {mchs }}$ sequence was more prevalent in laboratory strains compared to wildderived strains. Among 16 different tissues of $\sim 12$ week-old female C57BL/6J mice, brain homogenate was the only tissue with evident expression of ERV ${ }_{m c h 8}$. Further $E R V_{m c h 8}$ expression analysis in six different brain compartments and four peripheral neuronal tissues of C57BL/6J mice revealed no significant expression except for the cerebellum in which the ERV $V_{\text {mchs }}$ locus' low methylation status was unique compared to the other brain compartments. The

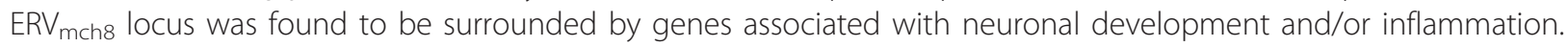
Interestingly, cerebellum-specific ERV ${ }_{\text {mchs }}$ expression was age-dependent with almost no expression at 2 weeks and a plateau at 6 weeks.
\end{abstract}

Conclusions: The ecotropic ERV $V_{\text {mchs }}$ locus on the C57BL/6J mouse genome was relatively undermethylated in the cerebellum, and its expression was cerebellum-specific and age-dependent.

\section{Background}

The concept of "endogenous" retroviruses (ERVs), which are inherited to subsequent generations by Mendelian order, was introduced following the discovery of three variants of ERVs in the genomes of laboratory mice and domestic fowls: murine leukemia virus (MuLV), mouse mammary tumor virus (MMTV), and avian leukosis virus [1,2]. ERVs are a family of long-terminal repeat (LTR) retrotransposons, and they occupy $~ 10 \%$ of the mouse genome $[3,4]$. In conjunction with the ERV population data accumulated from studies during the last few decades, the current mouse genome database renders an in-depth and systematic cataloguing of ERVs and other transposable and/or repetitive elements $[4,5]$.

\footnotetext{
* Correspondence: kcho@ucdavis.edu

'Department of Surgery, University of California, Davis, Sacramento, CA, USA Full list of author information is available at the end of the article
}

Mouse ERVs are segregated into three different classes (class I, II, III) based on the phylogenetic relatedness of their reverse transcriptase codons [6]. Class I (e.g., MuLV-type ERVs [MuLV-ERVs]), class II (e.g., MMTVtype ERVs), and class III ERVs represent $\sim 0.7 \%, \sim 3 \%$, and $\sim 5.4 \%$ of the mouse genome, respectively.

Some studies have shed an initial light into the biological properties of mouse ERVs. Rowe et al. reported that activation of recombinant MuLV-ERVs is linked to the onset of thymic lymphomagenesis [7]. In addition, it has been demonstrated that extended culturing of embryonic cells derived from certain mouse strains, such as AKR mice, resulted in the de novo production and release of MuLV-type ERVs [8,9]. Recent studies have suggested that the envelope gene products of ERVs participate in various pathophysiologic processes, such as placental morphogenesis in mice and demyelination 
of oligodendrocytes in multiple sclerosis patients $[10,11]$. Our laboratory reported that stress signals elicited from injury and/or infection activate certain ERVs, and lipopolysaccharide treatment differentially induces the production and release of ERV virions from mouse primary lymphocytes of various origins and at different developmental stages [12-14]. Furthermore, it was observed that ERV expression patterns in mice are directly linked to ERV-, cell-, and/or tissue-type [14,15].

In this study, using a combination of different ERV mining protocols, a full-length MuLV-ERV locus with an intact coding potential was identified from the C57BL/6J mouse genome. The genomic distribution of this ERV in different mouse strains and its expression characteristics in various tissues, including different brain compartments, were investigated.

\section{Results}

Identification of a full-length MuLV-ERV locus on chromosome 8 of the C57BL/6J mouse genome In our previous study, a stretch of 40 nucleotides at the junction of the envelope gene and 3' LTR of an unknown LTR retrotransposon was serendipitously identified during a genome-wide mining of MuLV-ERVs (Figure 1A) (unpublished). Using the 40 nucleotide sequence as an in silico probe, a combination of search programs, mainly NCBI BLASTN and BLASTP, was used to mine new ERV loci in the C57BL/6J mouse genome. Putative ERV loci identified from this mining experiment were subjected to an initial screening by an open reading frame (ORF) analysis and alignment against known ERVs. One putative full-length $(8,728$ nucleotides) MuLV-ERV was mapped on chromosome 8 (named "ERV ${ }_{\text {mch8 }}$ "), and it was determined to retain the intact coding potential for all three retroviral polypeptides (gag [537 amino acids], pro-pol [1,196 amino acids], and env [669 amino acids]) essential for virion assembly and replication (Figure $1 \mathrm{~B}, \mathrm{C}$ ). In addition, there were two identical LTRs of 523 nucleotides, a tRNA ${ }^{\text {Proline }}$ primer binding site, and an N-tropic motif in p30 of the gag gene on the ERV $\mathrm{mch}_{8}$ locus [16]. Phylogenetic analyses using three reference MuLV-ERVs (Emv1, MelRV, and NeRV), which share high sequence similarities with $E R V_{\text {mch8 }}$, revealed that $E R V_{\text {mch8 }}$ retains

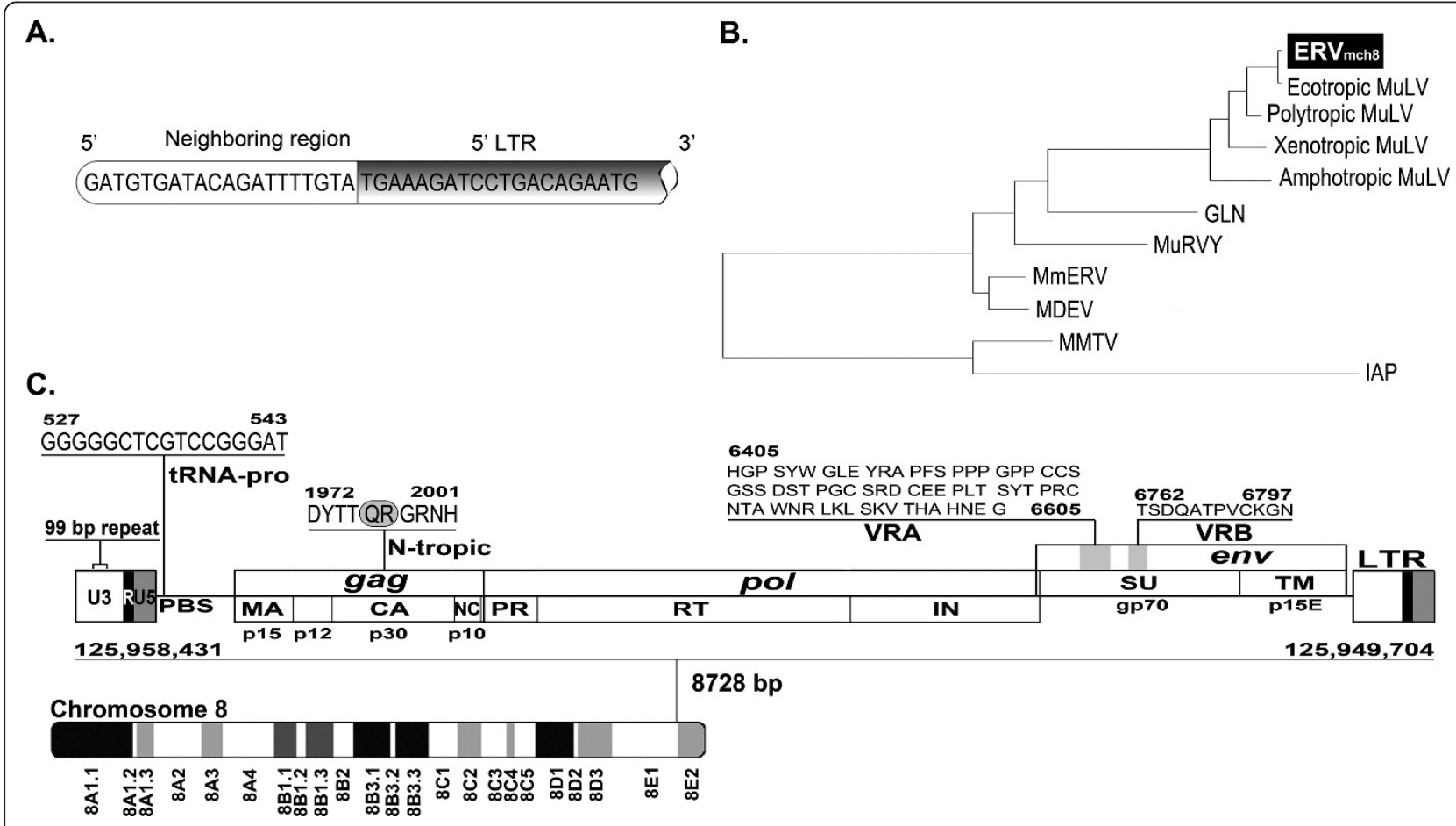

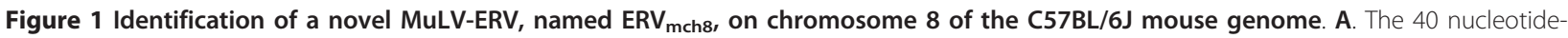
probe, which was serendipitously identified during an ERV survey experiment, was used to mine new ERVs from the NCBI C57BL/6J mouse

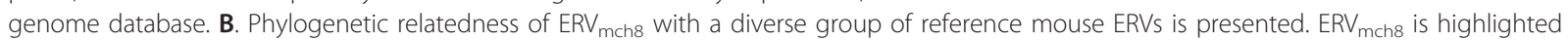
(black box). Reference ERVs: ecotropic MuLV (U63133.1), polytropic MuLV (U13766), xenotropic MuLV (DQ399707), amphotropic MuLV (AF411814.1), GLN (AC136922), MuRVY (X87639.1), MmERV (AC005743), MDEV (AF053745), MMTV (AF228550.1), and IAP (AB099818.1). C. The genomic location of ERV $\mathrm{mch}_{8}$ mapped to chromosome 8 of the C57BL/6J genome. Functional features (proviral size, primer binding site, tropism

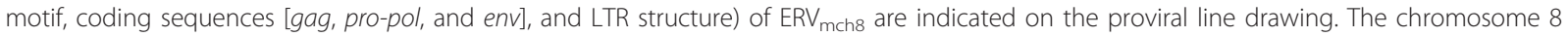
ideogram was adopted and modified from the NCBI mouse genome database. 
one polymorphic cluster in the gag gene (Figure 2) [17-19]. According to previous reports, it appears that ERV $V_{\text {mch8 }}$ shares the same genomic locus with another MuLV-ERV, called Emv2; however, this was not successfully confirmed because of an absence of relevant annotation and sequence information in the NCBI databases [20-22].

\section{Distribution of the $\mathrm{ERV}_{\text {mch8 }}$ sequence in the genomes of laboratory and wild-derived mouse strains}

To determine the distribution of the ERV $V_{\text {mch8 }}$ sequence in the genomes of laboratory and wild-derived mouse strains, genomic DNA samples isolated from 57 different strains were subjected to PCR genotyping using a primer set specific for the $E R V_{\text {mch8 }}$ sequence. The bands of the expected size were amplified in the vast majority of laboratory mouse strains, such as AKR/J and $\mathrm{C} 3 \mathrm{H} /$ $\mathrm{HeJ}$; conversely, they were present in only a limited number of wild-derived strains, such as MOLC/RkJ, MOLD/RkJ, and MOLF/EiJ (Figure 3A). The ERV $\mathrm{mch}_{\text {8 }}$ sequence was not amplified in the pahari/Ei and caroli/ EiJ strains, which are among the phylogenetically oldest wild-derived strains. Interestingly, the size and intensity of the bands, presumed to be amplified from the $E R V_{\text {mch8 }}$ sequences, were slightly variable depending on the mouse strain, suggesting polymorphisms in the sequences and/or copy numbers. Forty-seven of the 57 mouse strains were then mapped on Petkov et al.'s phylogenetic tree, which was established based on the profile of a set of single nucleotide polymorphism markers spanning the entire mouse genome, and is divided into seven distinct groups (Figure 3B) [23]. Interestingly, 16 of the 19 mouse strains mapped in Group 7 did not have evident amplification, whereas nine of the 11 in Group 1 as well as seven of eight in Group 4 had the expected bands (Figure 3).

\section{Brain-specific $\mathrm{ERV}_{\text {mch8 }}$ expression}

We then examined the expression pattern of $\mathrm{ERV}_{\text {mch8 }}$ in a set of 16 selected tissues from female C57BL/6J mice ( $\sim 12$ weeks-old). No significant levels of expression were observed in any tissues examined except for the brain homogenates (Figure 4A). It needs to be noted that the brain homogenates were prepared using half of a brain from each animal. The findings from this experiment led us to speculate that the expression of the $E R V_{\text {mch8 }}$ might be specific for certain compartment(s) of the brain and other neuronal tissues. In addition to the six discrete compartments of the brain (cerebral cortex, corpus callosum, brain stem, cerebellum, hippocampus, and olfactory bulb), cervical and lumbar spinal cords, optic nerve, and trigeminal ganglia were separately collected from female C57BL/6J mice ( 12 weeks-old) (Figure $4 \mathrm{~B}$ ) and were examined for the expression of
ERV $V_{\text {mch8. }}$ Interestingly, the evident expression of ERV $V_{\text {mch8 }}$ was detected only in the cerebellum (Figure $4 C)$. This cerebellum-specific pattern probably explains the variable expression levels of ERV $\mathrm{mch}_{\mathrm{mc}}$ in the brain homogenates processed from the half brains of three different mice, which may not represent the cerebellum proportionally (Figure 4A).

\section{Age-dependent regulation of the expression of $\mathrm{ERV}_{\text {mch8 }}$ in the cerebellum}

In this study, we examined whether the cerebellum-specific expression of $\mathrm{ERV}_{\mathrm{mch}}$ is developmentally regulated using six different brain compartments (cerebral cortex, corpus callosum, brain stem, cerebellum, hippocampus, and olfactory bulb) from eight different age groups of female C57BL/6J mice, ranging from $\sim 2$ to $\sim 29$ weeksold. No substantial expression of ERV $\mathrm{Em}_{\text {mch }}$ was noted in the cerebellum until four weeks of age, and the expression plateaued at $\sim 6$ weeks of age (Figure 5 ). In contrast, there was no evident expression of ERV $\mathrm{mch}_{\text {8 }}$ in the other brain compartments in all age groups examined. This finding suggests that the cerebellum-specific expression of $E R V_{\text {mch8 }}$ is age-dependent and potentially linked to the development of the cerebellum.

Protein coding sequences neighboring the $\mathrm{ERV}_{\text {mch8 }}$ locus The transcription regulatory elements residing on the ERV sequences may participate in modulating the expression of neighboring protein coding sequences $[24,25]$. The genomic regions surrounding the ERV $\mathrm{mch}_{\text {mo }}$ locus, $100 \mathrm{~Kb}$ upstream and $100 \mathrm{~Kb}$ downstream, were surveyed for annotated protein coding sequences on both strands. A total of eight protein coding sequences were identified: Spire2 (actin organizer), Tcf25 (transcription factor 25), Mc1r (melanocortin-1 receptor), Tubb3 (tubulin- $\beta 3$ ), Def8 (differentially expressed in FDCP 8), Afg3l1 (ATPase family gene 3-like 1), Dbndd1 (dysbindin domain containing 1), and Gas8 (growth arrest specific 8) (Figure 6). Interestingly, the majority of these protein coding sequences were characterized to be associated with neuronal development and/or inflammation [26-31]. For example, Tubb3 and Spire2 are involved in processes responsible for brain development, while Mc1r plays a role in brain inflammation [32,33]. Further studies may confirm the possibility that ERV $V_{\text {mch8 }}$ participates in the transcriptional control of some of these neighboring protein coding sequences.

\section{Unique methylation profile of the $\mathrm{ERV}_{\text {mchs }}$ locus in the cerebellum in comparison to the other brain compartments}

In this study, we attempted to determine whether the cerebellum-specific expression of $E R V_{\text {mch8 }}$ is linked to the methylation status of its cytosine residues. The 
A.

Full virus (nucleotide)

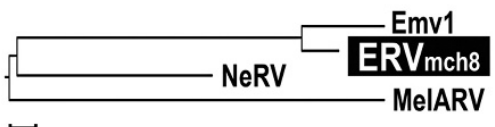

$\mathfrak{1 0}$ nucleotides substitution per 100 residues

pol (amino acid)

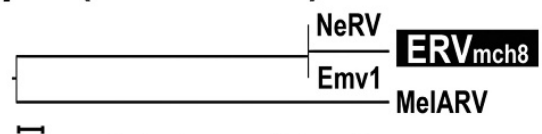

1 a.a. substitution per 100 residues

\section{gag (amino acid)}

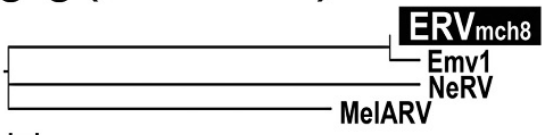

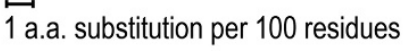

env (amino acid)

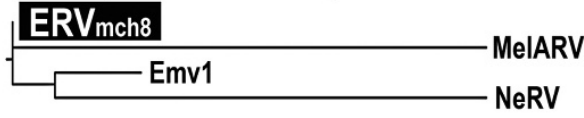

1a.a. substitution per 100 residues

B.

\section{Polymorphic gag region}

\begin{tabular}{|c|c|c|c|c|c|c|c|c|}
\hline \multirow{2}{*}{\multicolumn{9}{|c|}{ 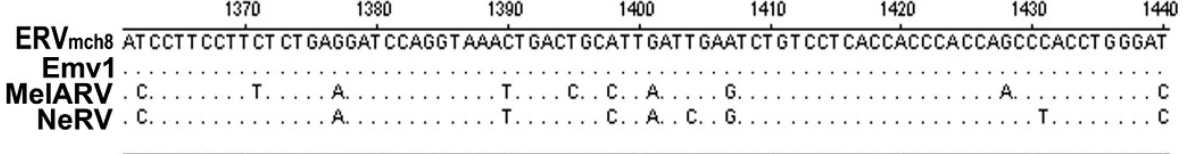 }} \\
\hline & & & & & & & & \\
\hline & 1450 & 1480 & 1470 & 1480 & 1480 & 1500 & 1510 & \\
\hline
\end{tabular}

ERV mch8 GATT GCCAGCAAT TATT TGGGACT CT GCTTACCGGGGAGGAGAAGCAGCGG GT GCT CCT GGAAGCCCGAAAAGCT GT CCG

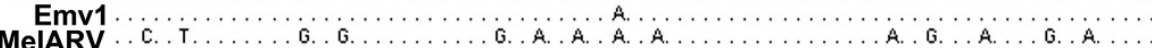

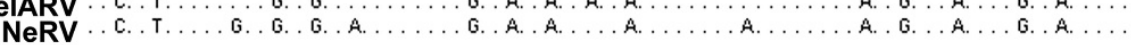

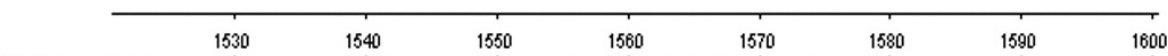

ERV mch $_{\text {GGGCAACGAT GGGCGCCCCACCCAACT GCCCAACGAGGTT GACGCT GCTTT TCCCCTT GAACGT CCCGAT G GGAT TACA }}$

Emv1

MelARV

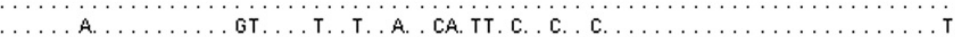

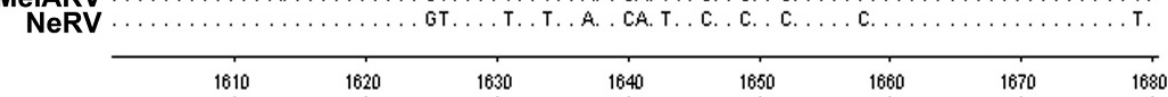

ERV ${ }_{\text {mch8 }}$ CCACCCAAAGAGGTAGGAACCACCT AGTTCT CTAT CGCCAGT T GCTTTTAGCGGGT CT CCAAAACGCGGGCCGAAGCCCC

Emv1

MelARV A. . . CTGA

\begin{tabular}{rrrrrrrr}
\hline 1690 & 1700 & 1710 & 1720 & 1730 & 1740 & 1750 & 1760
\end{tabular}

ERV mch8 ACCAATT T GGCCAAGGT AAAAGGAAT AACCCAGGGACCT GAT GAGT CCCCCT CAGCCT T CCT AGAGAGACT CAAGGAAGC

Emv1.

MelARV

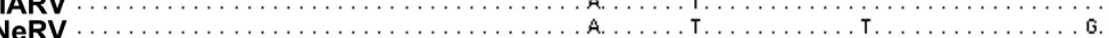

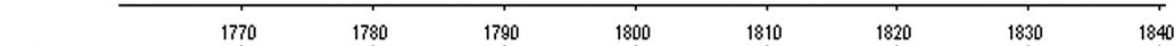

ERV ${ }_{\text {mch8 }}$ CTATCGCAGATACACT CCTTAT GAT CCT GAGGACCCT GGGCAAGAAACGAAT GTATCTAT GT CATTCATCT GGCAGTCCG Emv1

MelARV

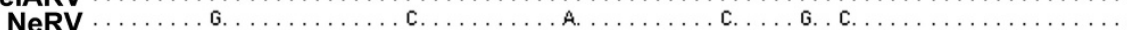

\begin{tabular}{rrrrrrr}
\hline 1850 & 1860 & 1870 & 1880 & 1890 & 1900 & 1910
\end{tabular}

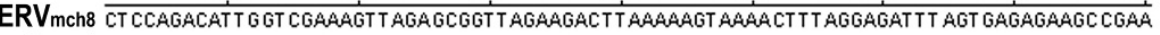

Emv1

MeIARV

NeRV

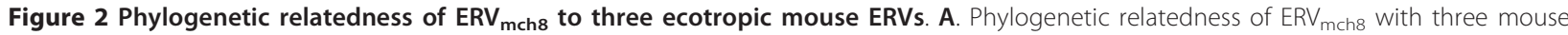
ecotropic ERVs, which have been characterized previously: complete nucleotide sequence (full provirus), gag polypeptide, pol polypeptide, and env polypeptide. MelARV (melanoma-associated retrovirus) (DQ366148.1), Emv1 (DQ366147.1), and NeRV (DQ366149.1) [17,18]. B. A main polymorphic region found in the gag p30 gene of ERV ${ }_{\text {mchs }}$ in comparison to the reference ecotropic ERVs is presented. Only the nucleotides different from the $\mathrm{ERV}_{\text {mchs }}$ sequence are indicated. 


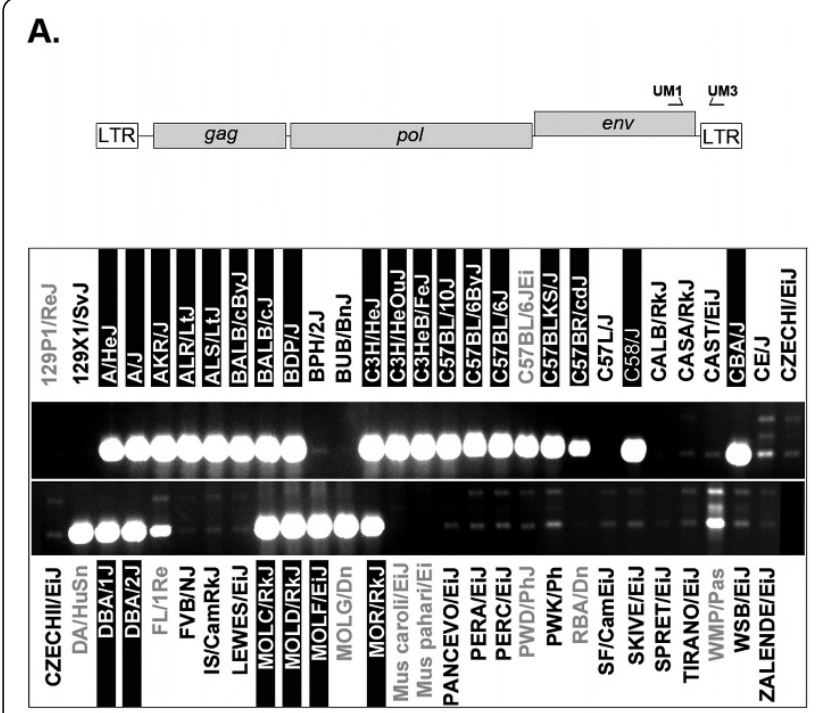

B.

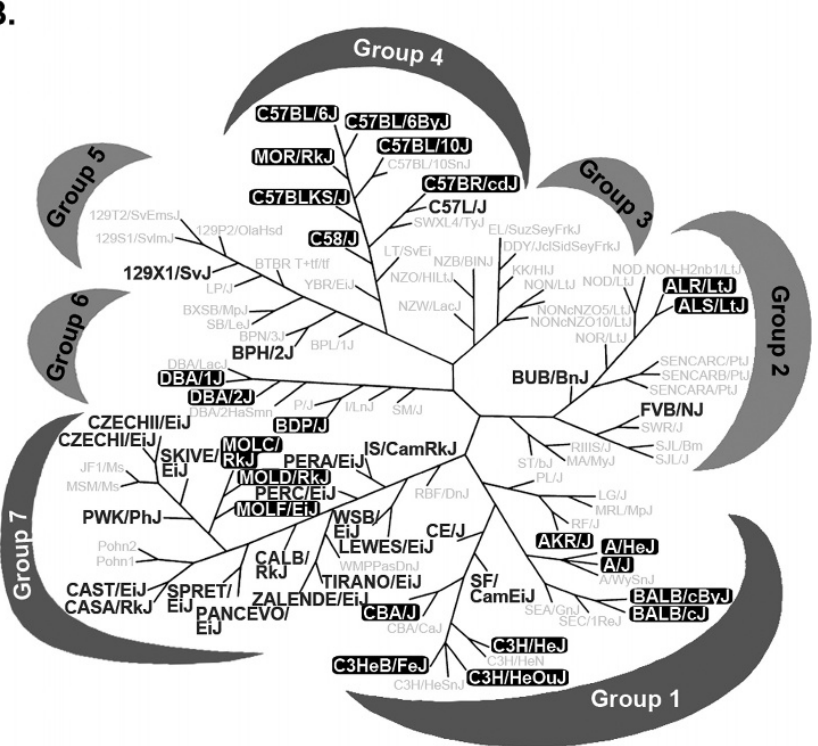

Figure 3 Distribution of the $\mathbf{E R V}_{\mathrm{mch} \mathbf{8}}$ sequence in $\mathbf{5 7}$ different mouse strains. A. A schematic drawing indicates the location of the primers

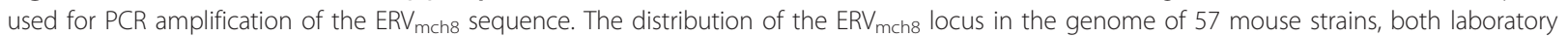
and wild-derived, was evaluated by PCR. B. The data regarding the distribution of the ERV $V_{\text {mchs }}$ sequence in various mouse strains are plotted onto a family tree developed by Petkov et al. [23]. Forty-seven of the 57 strains were mapped on the tree, which contains seven distinct groups,

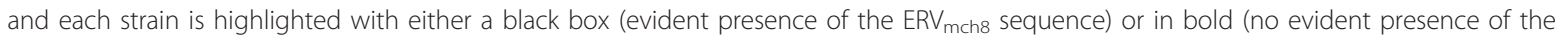
ERV $V_{\text {mch8 }}$ sequence).

methylation profile within a segment of the $E R V_{\text {mch8 }}$ provirus in the cerebellum, spanning the 3'-end of env gene to the U3 sequence, was compared to a group of five other brain compartments (brain stem, cerebral cortex, corpus callosum, hippocampus, and olfactory bulb) from $\sim 12$ week-old C57BL/6J mice. At numerous nucleotide positions for both strands, a significantly higher frequency of cytosine to thymine conversion was observed in the cerebellum in comparison to the rest of the brain compartments (Figure 7A). The cerebellum also had a unique profile of no conversion of cytosines in comparison to the other brain compartments. In the cerebellum, the number of nucleotide positions with a significant conversion frequency (red half-circle) was

\section{A.}

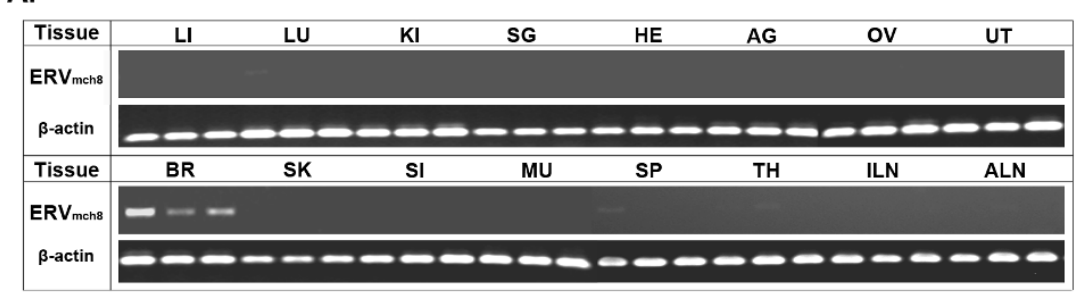

C.

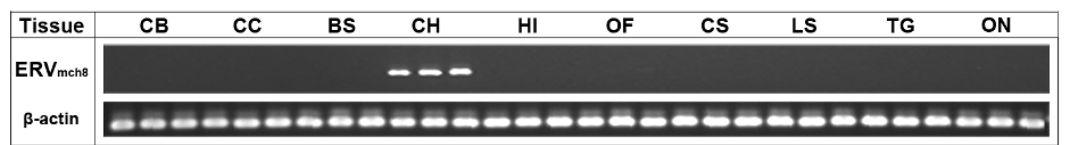

B.

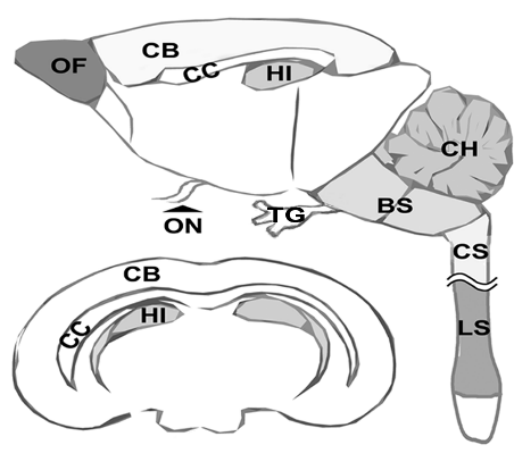

Figure 4 Cerebellum-specific ERV $_{\text {mch8 }}$ expression in C57BL/6J mice. A. Brain-specific expression of ERV $_{\text {mchs }}$ was identified by surveying 16 different tissues (liver [LI], lung [LU], kidney [KI], salivary gland [SG], heart [HE], adrenal gland [AG], ovary [OV], uterus [UT], brain homogenate $[\mathrm{BR}]$, skin $[\mathrm{SK}]$, small intestine $[\mathrm{SI}]$, muscle $[\mathrm{MU}]$, spleen $[\mathrm{SP}]$, thymus $[\mathrm{TH}]$, inguinal lymph node [ILN], and axillary lymph node [ALN]). $\beta$-actin serves as a normalization control. B. The drawing illustrates the relative locations of the brain compartments and other neuronal tissues (cerebral cortex $[\mathrm{CB}]$, corpus callosum $[\mathrm{CC}]$, brain stem $[\mathrm{BS}]$, cerebellum $[\mathrm{CH}]$, hippocampus [HI], olfactory bulb [OF], cervical spinal cord [CS], lumbar spinal cord [LS], trigeminal ganglia [TG], and optic nerve [ON]) examined in this study. C. Cerebellum-specific expression pattern of ERV $V_{\text {mchs }}$ was identified by the comparison of 10 different neuronal tissues. 


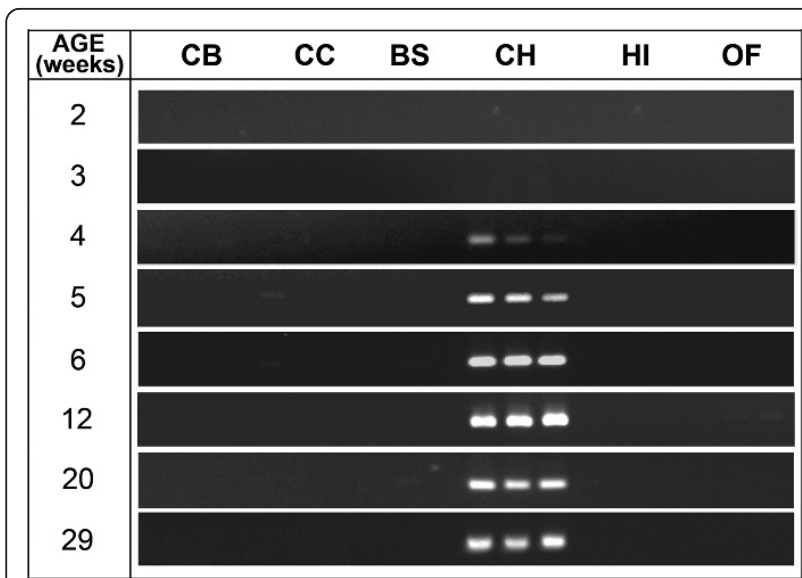

Figure 5 Age-dependent $\mathrm{ERV}_{\text {mch8 }}$ expression in the cerebellum Six different compartments of the brain (cerebral cortex [CB], corpus callosum [CC], brain stem [BS], cerebellum [CH], hippocampus [HI], and olfactory bulb [OF]) from eight different age groups of female C57BL/6J mice were surveyed for evidence of age-dependent/ developmentally-regulated expression of $\mathrm{ERV}_{\mathrm{mch}}$ in the cerebellum.

substantially higher than the positions with a significant no conversion frequency (blue half-circle): plus strand (46 conversion positions vs. 23 no conversion positions) and minus strand (75 conversion positions vs. 63 no conversion positions). In addition, the average number of converted cytosine residues, thus unmethylated, in the $E R V_{\text {mch8 }}$ sequences isolated from the cerebellum was significantly higher $(\mathrm{P}<0.01)$ compared to the rest of the brain compartments (Figure 7B). Furthermore, phylogenetic evaluation of the differentially converted $E R V_{\text {mch8 }}$ sequences was performed to compare the cytosine to thymine conversion profiles of the cerebellum and the other brain compartments (Figure 7C). Interestingly, the converted sequences isolated from the cerebellum formed a distinct branch for each strand: one branch had all seven plus strand sequences, and another branch contained all 12 minus strand sequences. Also, the converted sequences isolated from the brain stem were grouped into two small branches for each strand. The findings from these experiments suggest that the methylation profile of the $E R V_{\text {mch8 }}$ locus in the cerebellum is unique. Importantly, the number of unmethylated cytosine residues in the cerebellum was significantly higher compared to the rest of the brain compartments, which may be closely linked to the cerebellum-specific $\mathrm{ERV}_{\text {mch8 }}$ expression.

\section{Discussion}

Most characterized members of the C57BL/6J ERV population exist as multiple copies in the genome. A survey in this study identified only a single copy of an ecotropic ERV (ERV mch8$\left._{\text {8 }}\right)$ in the $C 57 \mathrm{BL} / 6 \mathrm{~J}$ mouse genome, and it is not currently annotated in the NCBI database (Build 37.1, as of November 12, 2010). ERV $_{\text {mch8 }}(8,728$ nucleotides) shares a greater than $98 \%$ nucleotide sequence homology with the melanomaassociated retrovirus (MelARV), which was localized on chromosome 7 of the B16 melanoma cell line derived from the C57BL/6 mouse strain [18]. According to the results obtained from the env polypeptide alignment against MelARV, it appears that ERV mch8 harbors an ecotropic tropism trait. Pothlichet et al. reported that a single locus on chromosome 8-qE1 was mapped using the MelARV env sequence as a query and presumed that MelARV originated from the Emv2 locus, which is reported to be the only ecotropic ERV found in normal C57BL/6 cells $[17,34]$. Contrary to this report, the ERV $V_{\text {mch8 }}$ locus, which has $\sim 98 \%$ nucleotide sequence homology with MelARV, was mapped on the chromosome 8-qE1 junction, based on survey results using NCBI BLAST. In addition, Emv2 is located/annotated at $67.0 \mathrm{cM}, \sim 11.4 \mathrm{cM}$ upstream of the ERV $\mathrm{mch}_{\text {8 }}$ locus $(\sim 78.4 \mathrm{cM})$, according to a survey of the NCBI map viewer $[21,35]$ http://www.ncbi.nlm. nih.gov/projects/mapview. Thus, it is probable that

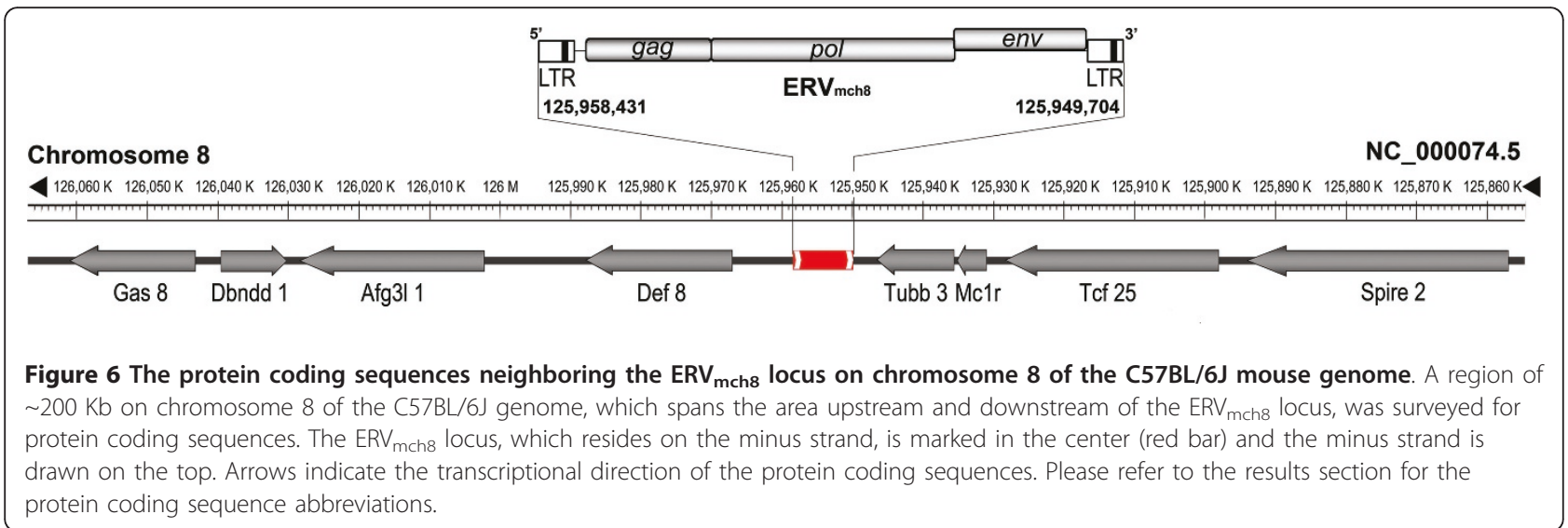




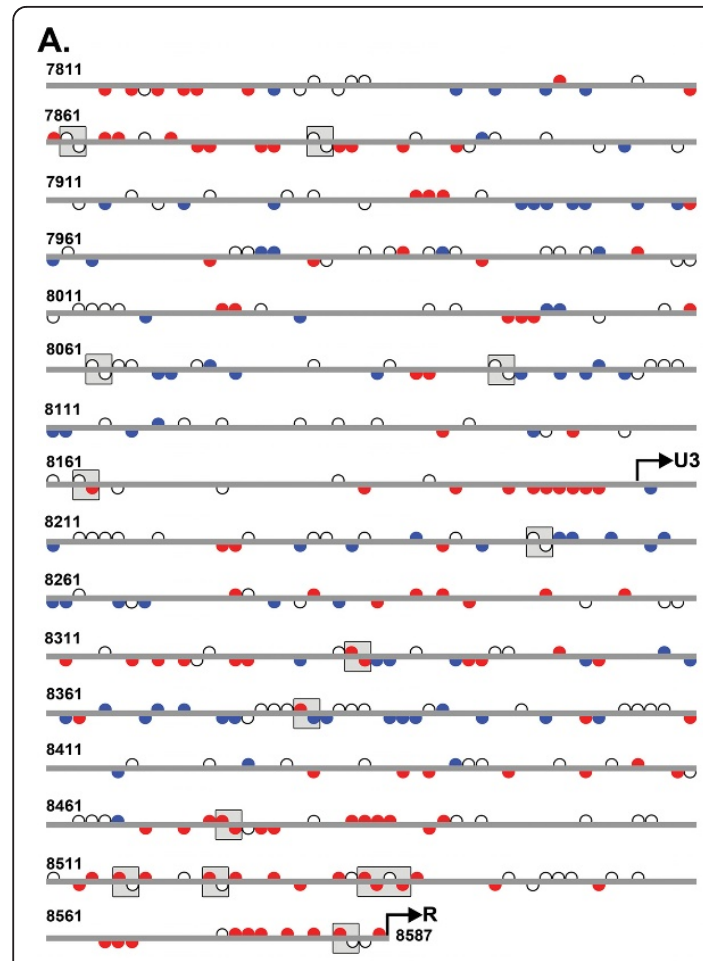

C.

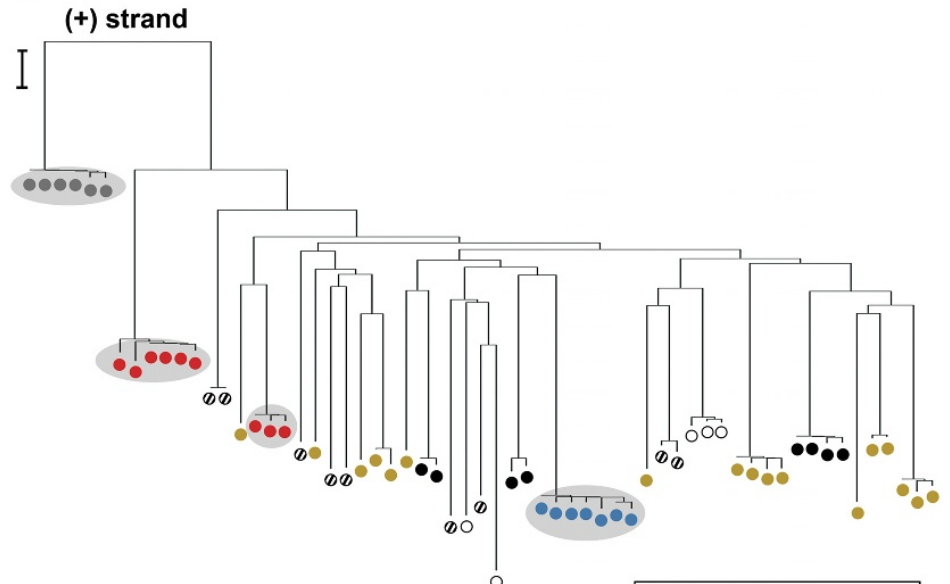

B.
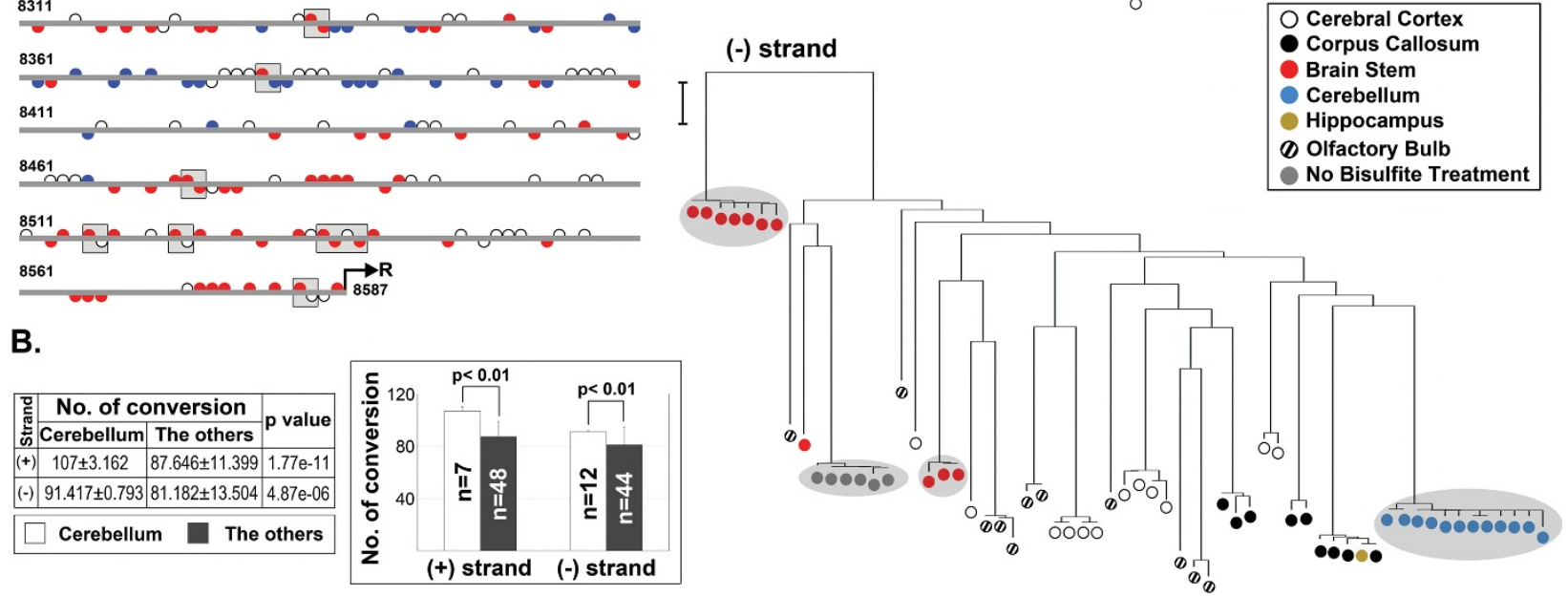

Figure 7 Cerebellum-specific methylation pattern of the ERV $_{\mathbf{m c h}}$ locus. A. The cytosine methylation profile of ERV $\mathrm{mchs}_{\mathbf{m}}$ in the cerebellum was compared to the methylation profile of an amalgamation of other brain compartments (cerebral cortex, corpus callosum, brain stem, hippocampus, and olfactory bulb). In particular, significant difference in the frequency of cytosine to thymine conversion (red half-circle) or in the frequency of no conversion (blue half-circle) in the cerebellum in comparison to the rest of the brain compartments is presented on both plus and minus strands. Open half-circle indicates no significant difference in the conversion frequency. Gray box identifies CpG dinucleotides. Start

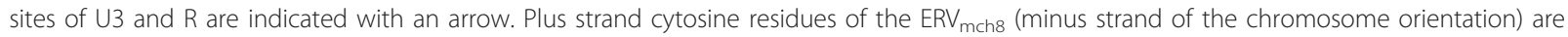
indicated by upward circles and minus strand with downward circles. B. The average number of converted cytosine residues in the ERV mch8 $_{2}$ sequences isolated from the cerebellum was compared to the other brain compartments; values are summarized in a table and presented as a bar graph. $\mathrm{N}$ (number of sequences analyzed). C. Phylogenetic evaluation of the differentially converted ERV $_{\text {mchs }}$ sequences was performed to compare the cytosine to thymine conversion profiles of the cerebellum and the other brain compartments. The bar on the left side of the tree represents the length of a nucleotide substitution per 100 residues.

ERV $V_{\text {mch8 }}$, but not Emv2, is the probable progenitor of MelARV, if any. Unexpectedly, we were unable to retrieve the nucleotide sequence, which is presumed to be the Emv2 provirus, from the Emv2 locus annotated in the NCBI C57BL/6J mouse genome (Build 37.1, as of November 12, 2010) and MGI (MGI_4.4 as of November 19, 2010) databases. Further, we were unsuccessful in locating the Emv2 proviral sequence, either partial or full, using the keyword, "Emv2", in the NCBI Nucleotide database. However, it is still a possibility that $\mathrm{ERV}_{\text {mch8 }}$ shares the same locus on chromosome 8-qE1 region with Emv2 with an assumption that the NCBI annotation information regarding the Emv2 locus needs to be revised.

Analysis of the distribution of the $\mathrm{ERV}_{\mathrm{mch}}$ sequence among various mouse strains demonstrated that a majority of strains in Groups 1 and 4 of the phylogenetic tree, which was developed by Petkov et al., harbor the proviral sequence in their genome. Within Group 1, which consists of mostly laboratory strains, including $\mathrm{BALB} / \mathrm{cJ}$ and $\mathrm{C} 3 \mathrm{H}$, all except for the SF/CamEiJ and $\mathrm{CE} / \mathrm{J}$ strains had evident amplification of the ERV $\mathrm{mch}_{\text {m }}$ sequence. The C57L/J strain in Group 4, which also contains the $\mathrm{C} 57 \mathrm{BL} / 6 \mathrm{~J}$ strain, did not have the $\mathrm{ERV}_{\text {mch8 }}$ 
sequence amplified, and this finding is consistent with the description from the Jackson Laboratory that "C57L/ $\mathrm{J}$ mice carry no detectable endogenous ecotropic MuLV DNA sequences". On the contrary, there was no amplification of the $E R V_{\text {mch8 }}$ sequence in the vast majority (16 of 19) of Group 7, which is comprised of wild-derived strains. Interestingly, a unique branch of three strains (MOLC/RkJ, MOLD/RkJ, and MOLF/EiJ) in Group 7, which had the ERV $V_{\text {mch } 8}$ sequence amplified, were derived by independent pairings of Mus musculus molossinus mice originating from Fukuoka, Japan (JAX ${ }^{\circledR}$ NOTES Issue 456 and JAX Mice Database, Jackson Laboratory). The SPRET/EiJ mice, also from Group 7 and derived from wild mice caught in Puerto Real, Spain (JAX Mice Database, Jackson Laboratory), had no ERV $V_{\text {mch8 }}$ sequence amplified. These findings suggest that the ERV $V_{\text {mch8 }}$ sequence is present in wild mice originating only from certain geographic regions.

The unique methylation profile, in particular, the high number of converted cytosines in a segment of the ERV $V_{\text {mch8 }}$ sequence of the cerebellum ( 12 week-old mice) in comparison to the other brain compartments, may explain, at least in part, the cerebellum-specific expression of the ERV $V_{\text {mch8 }}$ locus. Active transcription of this full-length $\mathrm{MuLV}-\mathrm{ERV}\left(\mathrm{ERV}_{\mathrm{mch}}\right)$, presumed to retain the ecotropic tropism trait, from the age of five to six weeks may lead to a series of potential short-term and long-term events: 1) persistent expression of gag, pol, and env polypeptides, and their potential contribution to the biology of the cerebellum, 2) assembly of virus particles with ecotropic tropism followed by their release, and 3) very low-level, if any, infection (due to presumed to be poor replication-competency) of neighboring and/or distant cells expressing relevant receptor (s) during the course of the relatively long lifespan of brain cells [36].

\section{Conclusions}

The key finding of this study that ERV $\mathrm{m}_{\text {mch8 }}$ expression is cerebellum-specific and age-dependent suggests that the expression of ERV $\mathrm{m}_{\mathrm{mch}}$ is linked to the biology of the cerebellum. A set of further experiments is needed to unveil the detailed mechanisms controlling the cerebellum-specific and age-dependent expression of ERV $\mathrm{mch}_{\text {m }}$ In addition, a full investigation into the roles of ERV $V_{\text {mch8 }}$ in the biology of the cerebellum and potentially other tissues is warranted.

\section{Methods}

\section{Animals}

Eight different age groups ( $\sim 2$ to $\sim 29$ weeks) of female C57BL/6J mice and $\sim 12$ week-old females were purchased from the Jackson Laboratory-West (West Sacramento, CA). The experimental protocol was approved by the Animal Use and Care Administrative Advisory Committee of the University of California, Davis. Three mice from each age group were sacrificed by $\mathrm{CO}_{2}$ inhalation or cervical dislocation followed by harvesting of different sets of tissues depending on the age groups. Certain brain samples were dissected further into their separate compartments and all tissue samples were snap-frozen.

\section{Genotyping PCR}

Genomic DNA samples from 57 different inbred mouse strains (both laboratory and wild-derived) were purchased from the Jackson Laboratory (Bar Harbor, Maine). Genotyping PCR was performed using $100 \mathrm{ng}$ of genomic DNA to determine the presence of $E R V_{\text {mch8 }}$ sequence using Taq polymerase from Qiagen (Valencia, CA) and a set of primers; UM1: 5'-GAA GTT GAA AAG TCC ATC ACT AA-3' and UM3: 5'-TCT GGG TCT CTT GAA ACT GT-3'.

\section{RNA isolation and RT-PCR}

Total RNA was isolated from the tissue samples using an RNeasy Lipid Tissue Mini Kit (for brain tissues) or RNeasy Mini Kit (for non-brain tissues) from Qiagen. cDNAs were synthesized from $100 \mathrm{ng}$ of total RNA using a QuantiTect Reverse Transcription Kit (Qiagen). A region near the 3 '-end of the $E R V_{\text {mch8 }}$ transcript was amplified by PCR using the UM1 and UM3 primer set (see above). $\beta$-actin served as a normalization control. Primers for $\beta$-actin amplification are as follows; Forward: 5'-CCA ACT GGG ACG TGG AA-3' and Reverse: 5'-GTA GAT GGG CAC AGT GTG GG-3'.

\section{Genomic DNA isolation, bisulfite treatment, and PCR amplification}

Genomic DNA was isolated from six different brain compartments (cerebral cortex, corpus callosum, brain stem, cerebellum, hippocampus, and olfactory bulb) of $\sim 12$ weeks-old mice using a DNeasy Tissue Kit (Qiagen). For the conversion of unmethylated cytosines to uracils/thymines, $2 \mu \mathrm{g}$ of genomic DNA from each sample was treated with bisulfite using a Methyl Detector Kit (Active Motif, Carlsbad, CA). PCR was performed using Taq polymerase (Qiagen), $7.5 \mu \mathrm{l}$ of bisulfite-treated DNA, and a set of primers; UM1 (see above) and m-L2D: 5'-CAA AAR RCT TTA TTR RAT ACA C-3'.

\section{Cloning and sequencing of PCR products}

PCR products were purified using a QIAquick Gel Extraction Kit (Qiagen) followed by cloning into the pGEM $^{\circledR}$-T Easy vector (Promega, Madison, WI). Plasmid DNA was prepared using a QIAprep Spin Miniprep Kit (Qiagen) for sequencing at Functional Biosciences (Madison, WI). 


\section{ERV mining, sequence alignment, and phylogenetic analyses}

The National Center for Biotechnology Information (NCBI) BLASTN and BLASTP programs were alternately used for mining new ERVs from the C57BL/6J mouse genome database with a 40 nucleotide probe, which was serendipitously identified in our previous study (unpublished). Alignment and phylogenetic analyses of the DNA, including the bisulfite-converted DNA clones, and protein sequences were performed using the MegAlign program from DNASTAR (Madison, WI).

\section{Statistical analyses}

The significance of differences in the $\mathrm{C}$ to $\mathrm{T}$ conversion rate at individual cytosine residue positions (plus and minus strands) was evaluated by Fisher's Exact probability test. The differences in the number of converted cytosine residues in the $E R V_{\text {mch8 }}$ sequence between the cerebellum and the other five brain compartments were evaluated by a Student's t-test. P values of less than 0.05 were determined to be significant.

\section{Acknowledgements and funding}

This study was supported by grants from Shriners of North America (No. 86800 to KC, No. 84302 to KHL [postdoctoral fellowship]) and the National Institutes of Health (R01 GM071360 to KC).

\section{Author details}

'Department of Surgery, University of California, Davis, Sacramento, CA, USA. ${ }^{2}$ Department of Neurology, University of California, Davis, Sacramento, CA, USA. ${ }^{3}$ Shriners Hospitals for Children Northern California, Sacramento, CA 95817, USA.

\section{Authors' contributions}

This study was conceived and managed by KC. DGG and TI participated in scientific discussions. $\mathrm{KHL}$ and $\mathrm{MH}$ performed the experiments and $\mathrm{KHL}$ generated the figures and drafted the manuscript. All authors read and approved the final manuscript.

\section{Competing interests}

The authors declare that they have no competing interests.

Received: 25 April 2011 Accepted: 12 October 2011

Published: 12 October 2011

\section{References}

1. Lupiani B, Hunt H, Silva R, Fadly A: Identification and characterization of recombinant subgroup J avian leukosis viruses (ALV) expressing subgroup A ALV envelope. Virology 2000, 276:37-43.

2. Weiss RA: The discovery of endogenous retroviruses. Retrovirology 2006, 3:67.

3. Smit AF: Interspersed repeats and other mementos of transposable elements in mammalian genomes. Curr Opin Genet Dev 1999, 9:657-663.

4. Waterston RH, Lindblad-Toh K, Birney E, Rogers J, Abril JF, Agarwal P, Agarwala R, Ainscough R, Alexandersson M, An P, et al: Initial sequencing and comparative analysis of the mouse genome. Nature 2002, 420:520-562.

5. Gibbs RA, Weinstock GM, Metzker ML, Muzny DM, Sodergren EJ, Scherer S, Scott G, Steffen D, Worley KC, Burch PE, et al: Genome sequence of the Brown Norway rat yields insights into mammalian evolution. Nature 2004, 428:493-521.
6. McCarthy EM, McDonald JF: Long terminal repeat retrotransposons of Mus musculus. Genome Biol 2004, 5:R14.

7. Rowe WP, Hartley JW: Genes affecting mink cell focus-inducing (MCF) murine leukemia virus infection and spontaneous lymphoma in AKR F1 hybrids. J Exp Med 1983, 158:353-364.

8. Rowe WP, Hartley JW, Lander MR, Pugh WE, Teich N: Noninfectious AKR mouse embryo cell lines in which each cell has the capacity to be activated to produce infectious murine leukemia virus. Virology 1971, 46:866-876.

9. Aaronson SA, Hartley JW, Todaro GJ: Mouse leukemia virus: "spontaneous" release by mouse embryo cells after long-term in vitro cultivation. Proc Natl Acad Sci USA 1969, 64:87-94

10. Antony JM, Ellestad KK, Hammond R, Imaizumi K, Mallet F, Warren KG, Power $C$ : The human endogenous retrovirus envelope glycoprotein, syncytin-1, regulates neuroinflammation and its receptor expression in multiple sclerosis: a role for endoplasmic reticulum chaperones in astrocytes. J Immunol 2007, 179:1210-1224.

11. Gong R, Huang L, Shi J, Luo K, Qiu G, Feng H, Tien P, Xiao G: Syncytin-A mediates the formation of syncytiotrophoblast involved in mouse placental development. Cell Physiol Biochem 2007, 20:517-526.

12. Cho K, Adamson LK, Greenhalgh DG: Induction of murine AIDS virusrelated sequences after burn injury. J Surg Res 2002, 104:53-62.

13. Cho K, Lee YK, Greenhalgh DG: Endogenous retroviruses in systemic response to stress signals. Shock 2008, 30:105-116.

14. Kwon DN, Lee YK, Greenhalgh DG, Cho K: Lipopolysaccharide stress induces cell-type specific production of endogenous retroviral virions in primary lymphoid cells. J Gen Virol 2010, 92:292-300.

15. Kwon DN, Greenhalgh DG, Cho K: Cloning and characterization of endogenous retroviruses associated with postinjury stress signals in lymphoid tissues. Shock 2009, 32:80-88.

16. Ou CY, Boone LR, Koh CK, Tennant RW, Yang WK: Nucleotide sequences of gag-pol regions that determine the Fv-1 host range property of BALB/C $\mathrm{N}$-tropic and B-tropic murine leukemia viruses. J Virol 1983, 48:779-784.

17. Pothlichet J, Heidmann T, Mangeney M: A recombinant endogenous retrovirus amplified in a mouse neuroblastoma is involved in tumor growth in vivo. Int J Cancer 2006, 119:815-822.

18. Pothlichet J, Mangeney $M$, Heidmann T: Mobility and integration sites of a murine C57BL/6 melanoma endogenous retrovirus involved in tumor progression in vivo. Int J Cancer 2006, 119:1869-1877.

19. Poste G, Doll J, Brown AE, Tzeng J, Zeidman I: Comparison of the metastatic properties of B16 melanoma clones isolated from cultured cell lines, subcutaneous tumors, and individual lung metastases. Cancer Res 1982, 42:2770-2778.

20. Ihle JN, Joseph DR: Genetic analysis of the endogenous $\mathrm{C} 3 \mathrm{H}$ murine leukemia virus genome: evidence for one locus unlinked to the endogenous murine leukemia virus genome of C57BL/6 mice. Virology 1978, 87:298-306.

21. Ceci JD, Justice MJ, Lock LF, Jenkins NA, Copeland NG: An interspecific backcross linkage map of mouse chromosome 8. Genomics 1990, 6:72-79.

22. Jenkins NA, Copeland NG, Taylor BA, Bedigian HG, Lee BK: Ecotropic murine leukemia virus DNA content of normal and lymphomatous tissues of BXH-2 recombinant inbred mice. J Virol 1982, 42:379-388.

23. Petkov PM, Ding Y, Cassell MA, Zhang W, Wagner G, Sargent EE, Asquith $S$, Crew V, Johnson KA, Robinson P, Scott VE, Wiles MV: An efficient SNP system for mouse genome scanning and elucidating strain relationships. Genome Res 2004, 14:1806-1811.

24. Ting CN, Rosenberg MP, Snow CM, Samuelson LC, Meisler MH: Endogenous retroviral sequences are required for tissue-specific expression of a human salivary amylase gene. Genes Dev 1992, 6:1457-1465

25. Dunn CA, Medstrand P, Mager DL: An endogenous retroviral long terminal repeat is the dominant promoter for human beta1,3galactosyltransferase 5 in the colon. Proc Natl Acad Sci USA 2003, 100:12841-12846.

26. Schumacher N, Borawski JM, Leberfinger CB, Gessler M, Kerkhoff E: Overlapping expression pattern of the actin organizers Spir-1 and formin-2 in the developing mouse nervous system and the adult brain. Gene Expr Patterns 2004, 4:249-255.

27. Lasaga M, Debeljuk L, Durand D, Scimonelli TN, Caruso C: Role of alphamelanocyte stimulating hormone and melanocortin 4 receptor in brain inflammation. Peptides 2008, 29:1825-1835. 
28. Singh KK, Tsai LH: MicroTUB(B3)ules and brain development. Cell 2010 140:30-32.

29. Zheng Y, Cheng XR, Zhou WX, Zhang YX: Gene expression patterns of hippocampus and cerebral cortex of senescence-accelerated mouse treated with Huang-Lian-Jie-Du decoction. Neurosci Lett 2008, 439:119-124.

30. Kremmidiotis G, Gardner AE, Settasatian C, Savoia A, Sutherland GR, Callen DF: Molecular and functional analyses of the human and mouse genes encoding AFG3L1, a mitochondrial metalloprotease homologous to the human spastic paraplegia protein. Genomics 2001, 76:58-65.

31. Ghahramani Seno MM, Hu P, Gwadry FG, Pinto D, Marshall CR, Casallo G, Scherer SW: Gene and miRNA expression profiles in autism spectrum disorders. Brain Res 2010, 1380:85-97.

32. Tischfield MA, Baris HN, Wu C, Rudolph G, Van Maldergem L, He W, Chan WM, Andrews C, Demer JL, Robertson RL, Mackey DA, Ruddle JB, Bird TD, Gottlob I, Pieh C, Traboulsi El, Pomeroy SL, Hunter DG, Soul JS, Newlin A, Sabol LJ, Doherty EJ, de Uzcategui CE, de Uzcategui N, Collins ML, Sener EC, Wabbels B, Hellebrand H, Meitinger T, de Berardinis T, et al: Human TUBB3 mutations perturb microtubule dynamics, kinesin interactions, and axon guidance. Cell 2010, 140:74-87.

33. Ignar DM, Andrews $J$, Jansen $M$, Eilert MM, Pink HM, Lin P, Sherrill RG, Szewczyk JR, Conway JG: Regulation of TNF-alpha secretion by a specific melanocortin-1 receptor peptide agonist. Peptides 2003, 24:709-716.

34. Jenkins NA, Copeland NG, Taylor BA, Lee BK: Organization, distribution, and stability of endogenous ecotropic murine leukemia virus DNA sequences in chromosomes of Mus musculus. J Virol 1982, 43:26-36.

35. Acar H, Copeland NG, Gilbert DJ, Jenkins NA, Largaespada DA: Detection of integrated murine leukemia viruses in a mouse model of acute myeloid leukemia by fluorescence in situ hybridization combined with tyramide signal amplification. Cancer Genet Cytogenet 2000, 121:44-51.

36. Falk A, Frisen J: New neurons in old brains. Ann Med 2005, 37:480-486.

doi:10.1186/1742-4690-8-82

Cite this article as: Lee et al:: Cerebellum-specific and age-dependent expression of an endogenous retrovirus with intact coding potential. Retrovirology 2011 8:82.

\section{Submit your next manuscript to BioMed Central and take full advantage of:}

- Convenient online submission

- Thorough peer review

- No space constraints or color figure charges

- Immediate publication on acceptance

- Inclusion in PubMed, CAS, Scopus and Google Scholar

- Research which is freely available for redistribution

Submit your manuscript at www.biomedcentral.com/submit 